\title{
Using Association Rules for Energy Conservation in Wireless Sensor Networks
}

\author{
Suan Khai Chong ${ }^{1}$, Shonali Krishnaswamy ${ }^{1}$, Seng Wai Loke ${ }^{2}$, Mohamed Medhat Gaber ${ }^{3}$ \\ ${ }^{1}$ Monash University \\ 900 Dandenong Road \\ Caulfield, Australia \\ Suan.Chong, \\ Shonali.Krishnaswamy \\ ${ }^{2}$ Latrobe University \\ 215 Franklin Street \\ Melbourne, VIC 3000 , \\ Australia \\ @infotech.monash.edu.au \\ ${ }^{3}$ CSIRO ICT Centre \\ Hobart, Tasmania \\ Mohamed.Gaber \\ @ csiro.au
}

\begin{abstract}
Frequent radio transmissions among sensors, or from sensors to the basestation, have always been a major energy drain. One of the approaches to reduce the data transmitted to the basestation is to shift the bulk of data processing to networked sensor nodes; for instance, sensors to send only data aggregates to reduce the overall amount of data exchanged. Sensor nodes, however, are quite limited in terms of their energy and processing power, and as such, traditional centralised data mining algorithms are infeasible to be directly implemented on sensors. In this paper, we modify APRIORI to find strong rules from sensor readings in a sensor network and using these rules, autonomously control sensor network operations or supplement sensor operations with a rule knowledge base. For example, triggers activated from the rules could be used to sleep sensors or reduce data transmissions to conserve sensor energy. Our work here includes a detailed implementation of a lightweight rule learning algorithm for a resource-constrainted sensor network, with simulation results for a group node setup running the algorithm.
\end{abstract}

\section{INTRODUCTION}

A wireless sensor network (WSN) is described as a set of nodes capable of sensing, performing data computations and communicating data with one another wirelessly [3]. One class of inexpensive sensors that falls into this study is Berkeley motes [1], whereby a network could be formed by Mica2/Mica2dots equipped with MTS310 sensor boards, enabling the collecting of light, temperature, sound and motion readings. However, as observed in several applications, WSN deployments are often faced with unique challenges due to sensors' short battery lifespan. Furthermore, a widescale deployment of sensors in harsh environments make battery replacement a difficult task.

A way to prolong sensor battery life is by reducing radio transmissions over the network. As shown in [16], network radio transmission is the biggest factor of energy consumption by sensor nodes.

Permission to make digital or hard copies of all or part of this work for personal or classroom use is granted without fee provided that copies are not made or distributed for profit or commercial advantage and that copies bear this notice and the full citation on the first page. To copy otherwise, to republish, to post on servers or to redistribute to lists, requires prior specific permission and or a fee.

SAC'08 March 16-20, 2008, Fortaleza, Ceará, Brazil

Copyright 2008 ACM 978-1-59593-753-7/08/0003 ...\$5.00.
By shifting the bulk of the processing into networked sensor nodes, it allows nodes to send less data either to a neighbouring node or to a base-station. Nevertheless, it is important to note that sensors such as Berkeley motes have neither a hardware floating point unit nor sufficient memory to run complex data mining algorithms, such as k-means clustering or a Fast Fourier Transform algorithm. This would deplete sensor resources quickly. In this context, there have been recent work of some in-network processing algorithms for sensor network (refer \$2). Other related areas include Data Stream Management Systems(DSMS) such as [13] which have enabled queries to be filtered to send only data most relevant to the user and in [8], to predict sensor missing values or values of neighbouring sensors.

However, while previous approaches have tried to reduce the amount of data that needs to be transmitted in the network, we believe that learnt information can be utiltised more effectively by mining for highly correlated rules in the network and applying them as rule triggers on sensors directly to conserve their energy. As a motivating example, if after a period of sensing, a rule is discovered such that the sensory temperature is high for a certain period of the day when the light reading is low, then a trigger could be initiated to command antecedent sensors of the rule to send only the light reading, whereas the temperature reading will be inferred at the sink by consulting the rule repository.

In [6], such patterns can be termed more generally as contextual information in the sensor network and has been shown to achieve considerable energy savings for sensor networks offline. Motivated by our previous results, we extend our work to perform rulelearning on sensor streams to mine for such patterns and from the potential rules discovered, trigger sensor operations that conserve sensor energy. This approach is instantiated with an implementation of our customised APRIORI[2] to work on sensor nodes and early simulation results for a group working model.

The rest of this paper is organised in the following way. In section 2, we present an overview of past and present research in relation to our work. The data model for the system is described in section 3 . Section 4 details the algorithm for our approach. To validate our methods, we perform simulations using the algorithm on correlated sensor streams and record our observations in section 5 . Section 6 records proposed future work.

\section{RELATED WORK}

As an overview, [12] describes their study on the relevance of finding patterns in WSNs, particularly in terms of detecting sensor data irregularities, clustering of sensor data and discovery of sensor 
data correlations. Discovery of such patterns as decribed in their application to a panda habitat, would show us various information such as abnormal panda behaviour through outliers or correlations through finding the attributes of pandas that are correlated. In a similar context, spatio-temporal correlations have been explored in [8] where sensors can utilise correlations in data to predict their neighbours' readings. This motivates a need to further utilise strong correlations to conserve sensor energy.

On the other hand, several research projects in sensor networks have looked at ways to minimise sensor network energy consumption. At the hardware level, these approaches have included lowpower design techniques for sensors as described in [5] and costefficient hardware such as low-power wakeup radios [10]. However, while hardware-based power saving techniques have been beneficial to reducing the energy consumption of single sensor nodes, such approaches are limited to specific sensor hardware. Conserving energy in a wireless sensor network extends to maximising the lifetime of the whole sensor network.

Explained in [16], this entails embodying energy awareness into every stage of a wireless sensor network design and operation. As such, numerous authors including $[4,18]$ have explored issues in sensor network designs that could improve energy efficiency of the whole system. Generally, the targeted design issues have covered network topology, node communication strategies, energy management and node failure recoveries; all of which are necessary for allowing sensor networks to be adjustable to their environment for maximal energy efficiency. A more specific example would be the multi-tier sensor network architecture proposed in [17], where entities having more resources in the network such as gateways are used to analyse sensor energy reports, monitor the status of sensors and control sensor operations using that information; for instance, a more efficient routing decision based on sensor energy levels. These studies, nevertheless, points us to distributing processing to the sensor network or the benefits of manipulating a heterogeneous sensor network.

The work most closely related to ours is [11]. In their work, they analysed the representation of a sensor data stream for mining and proposed an interval-list representation. Lossy-Counting is then performed on transactions collected using this data representation with favourable results using synthetic data sets. In terms of data analysis, we share a common ground on the use of support and confidence measure. We differ, however, on the data representation level for our purpose of mining for rules more efficiently on sensor nodes with multivariate data. We also believe that energy could be conserved through a rule analysis on the sensor notes.

Finding interesting correlations in sensor data constitutes part of our rule-mining algorithm. In [14], the authors investigated correlations that can be formed when sensors in loading truck experience similar vibrations when the trucks send out the same load. The correlation information of the sensor nodes then allowed them to group trucks carrying out the same load. Condensing parts of the earlier two approaches into our work, in the following sections, we discuss our own approach to conserve energy with highly correlated rules that can lead to triggers at sensor-level.

\section{DATA MODEL}

Our initial aim is to mine for correlations among pieces of sensor data. In our definition, a data correlation in sensor networks is a statistical interdependence between any variable sensor values in $\left\{a_{1}, a_{2}, \ldots, a_{n}\right\}$ where $a_{n}$ can be any possible sensed data sample for sensors $S_{1}, S_{2}, \ldots, S_{n}$. Let us assume we have sensors $S_{1}$, $S_{2}, S_{3}$ collecting sound $s$, temperature $t$ and light $l$ samples. The light reading $l_{1}$ of $S_{1}$ might be similar to $l_{2}$ of $S_{2}$ because they are detecting readings in the same area.

Typically, data sampled such as $t_{1}$ or $l_{1}$ is continuous data and would arrive in a random manner. As sensors are typically resourceconstrained with limited processing capabilities, it is infeasible for sensors to perform intensive computations on raw sensor data. To generate rules relevant for our purpose, we choose to consider only discretised sensor values, where the range is pre-defined. For example, for a light reading in $[0,1000]$, we assign states ' $L$ ' for readings in range $[0,299]$, ' $\mathrm{M}$ ' for readings in range $[300,699]$ and ' $\mathrm{H}$ ' for readings in range $[700,1000]$. On the second note, the arrival time of sensor data value sets is normally random. Thus, we model data transactions as data value sets $\left\{S_{n} ; t_{n}, s_{n}, l_{n}\right.$, time $\}$ in packet format, where time can be the number of minutes from a landmark time or the actual time that it arrives from a sensor (Refer [11] for the data arrival figure that we are modeling on).

A direct application of a rule mining algorithm such as APRIORI on sensors would require the generation of $k$ itemsets from the $k-1$ itemsets that are frequent, creating too many rules, which is infeasible for sensor nodes. Instead, in our work, we propose instead to count the number of transactions that are frequent, omitting the generation of any $k$ itemsets and also concentrate on only highly correlated rules. This is contrary to the weighted transformation method as used in [11] where itemsets are generated. We assume that transactions are processed in batches $b_{1}, b_{2}, \ldots, b_{x}$ where $1<x<k$ and $k<$ number of transactions in $b_{x}$, and that $x$ must also be sufficiently large as transactions cannot occur with equal probabilities (i.e. each transaction of support 1).

Therefore, for a batch $b_{x}$, the support of any transaction $n$, with all elements considered, is simply:

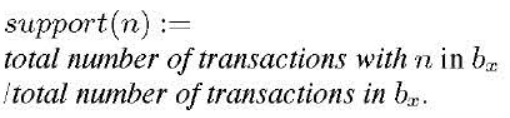

Subsequently, the confidence of a rule, for instance, $\left(S_{1}\right.$ temperature $\rightarrow S_{1}$ light $)$

i.e., $a_{n} \rightarrow a_{n-1}$, generated from a transaction over a user-defined support is given by

confidence $\left(a_{n}, a_{n-1}\right):=$ $\operatorname{support}\left(a_{n}, a_{n-1}\right) / \operatorname{support}\left(a_{n}\right)$

The confidence measure allows us to trigger rules as long as the premises hold. For instance, if we have a high confidence for a rule stating that $a_{n}$ implies $a_{n-1}$, the rule is extracted and we send only reading $a_{n}$ to the base-station/central node. Upon receiving the reading $a_{n}$ and utilising knowledge of the rule, the reading of $a_{n-1}$ can be inferred.

\section{ALGORITHM}

In this section, we describe the algorithm for mining rules from sensor data packets arriving at node $M$. In our later experiments, the rules discovered could then be used for a singular sensor to control its operations or used to transmit less packets given a query to the aggregate node.

With reference to Algorithm 1, we further explain the steps through this algorithm as follows:

Step $1 S$ is made up of heterogeneous sensors that form a group. Transaction batches are collected at a sensor node. Each sensor in $\mathrm{S}$ has one or more sensor attributes in $A$.

Step 2 Each sensor in $S$ has a finite amount of energy that can be derived from packets the sensors send. Thus, $e$ is in the attribute set $A$. We can assume that we dynamically update an energy map with new sensor readings. 


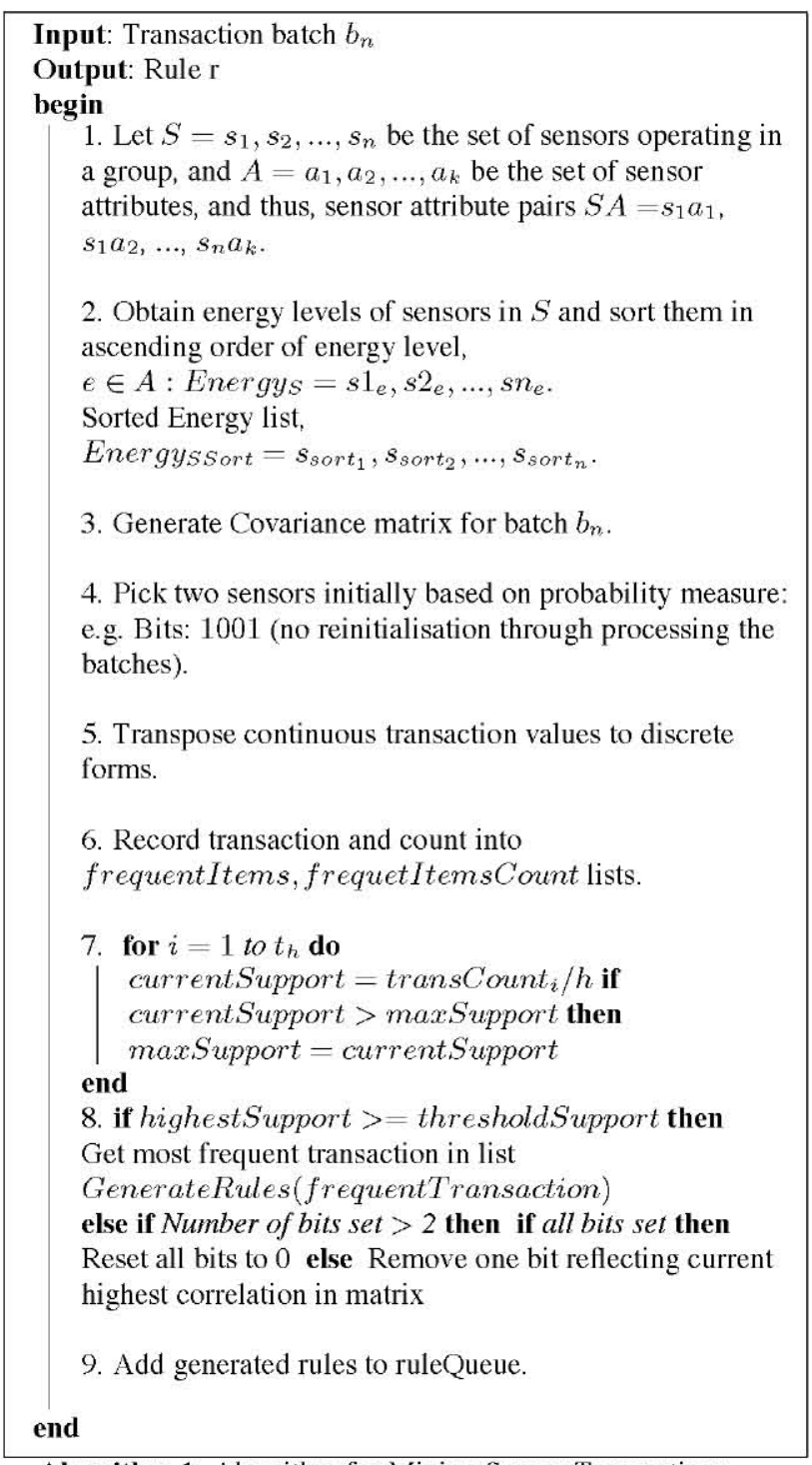

Algorithm 1: Algorithm for Mining Sensor Transactions

Step 3 For batch $b_{n}$, generate the the coefficient matrix $M$ for a maximum of $n * k$ rows by $n * k$ columns for sensors and their attributes. The correlation is calculated based on the current numerical values of the sensor attribute values. Formula used is:

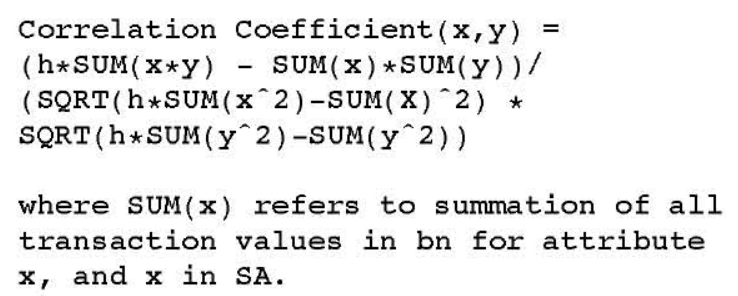

We can assume that this preprocessing step is done on a subset of the data rather than the whole dataset. Also, assume that this correlation matrix is valid for a time window long enough to ignore this possible inefficiency.
Step 4. When counting transactions, we only like to regard attribute combinations that contain sensors with high correlation values in $M$ and equal combination of sensors with low/high energy in Energy $S_{\text {sort }}$

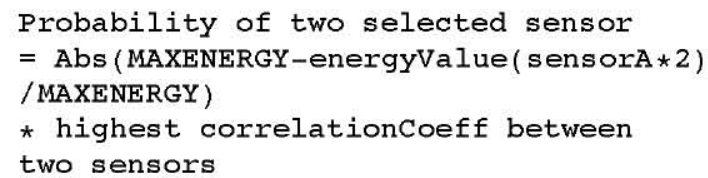

The formula allows us to choose sensors with the biggest difference in their energy levels and highest correlation coefficient.

The logic in choosing a transaction combination with biggest difference in energy levels is so that rules that are derived from the algorithm would form a trigger that could maximise the lifespan of our sensor network. In general, the rule with the lowest number of high energy sensors and highest number of low energy sensors has the best combination. For example, in a rule that has $S 1 \& S 2 \rightarrow S 3, S 3$ 's reading is being implied by both $S 1$ and $S 2$ 's readings. $S 1$ and $S 2$ are controlling sensors and thus will consume energy whereas $S 3$ 's energy is conserved. Thus, if $S 3$ is a dying sensor/low resources, we minimise it's energy use and extend the life of the network. Preference is hence given to such combinations.

On the other hand, we are also interested in not just any rule derived from the algorithm. We generally want rules with a high support and confidence, i.e. given $A \& B \rightarrow C$, a high likelihood of $C$, given $A$ and $B$. In the same way as the logic above, this is so that the trigger to be generated can rely on $S 1$ and $S 2$ 's reading to predict $S 3$. Pearson's product moment correlation coefficient gives us the measure of tendency of the variables to increase or decrease together. The preprocessing of transactions in this manner increases the quality of the rules obtained.

Given $k$ is the maximum number of attributes that any sensor in $S$ would have, we initialise $k$ number of bits to zero, and transaction attributes with bits set to zero won't be regarding in the counting and vice versa.

At initialisation, the algorithm would need to have at least two highly correlated attributes and sensors that come from both end of the energy spectrum.

Subsequently, extra bits that would be added would need to have a high correlation coefficient with bits existing in the bits array.

Bits array are reset to two again when all bits have been set.

Step 5 Numerical values of the sensor attributes are required at the preprocessing step of the algorithm. Following the preprocessing, we would convert all numerical values for individual sensors to discrete values so as to generate rules in value ranges only and to reduce complexity of the algorithm.

We use IF-ELSE statements to give discrete values to attribute ranges. An alternative is to autonomously determine the discrete states by classifying the values.

Step 6 A list of frequentItems and frequentItemsCount is maintained. This list is updated for every transaction in the batch. The frequentItems list stores the most frequent transactions in order and their corresponding counts in frequentItemsCount. The sizes of frequentItems list and frequentItemsCounts list are user-defined. frequentItems list is a $2 \mathrm{D}$ array with a user-defined number of items but with transaction size $>=2$ and $<=\mathrm{k}$.

Step 7 Get the highest support from transactions already in list.

Step 8 Check if the current transaction in batch has support greater than the threshold. Note that support is calculated from using the frequentItems list and frequentItemsCount list. We maintain a record of all transactions with support greater than 
threshold. If the support within the batch is greater than the threshold, generate the rules from this transaction. Set one more bit in the bits array in accordance with the probability measure. The rational for doing this is so that rules that will be generated next will hopefully involve more sensors in the same grouping that has met the threshold to conserve more energy.

If the threshold is not met, reduce number of bits set by one.

Step 9 After the rule is generated, it is added to a ruleQueue if it is above the threshold confidence. The ruleQueue is served periodically per user-defined intervals. Preliminary, we can rank the rules using the confidence of the rule.

Out of our rules, we then make a hashtable for the list of sensors to monitor with their expected values. At runtime, we periodically call a sensor routine to pick $k$ random readings from the current batch to compare sensors' readings with their expected values according to the list. If the values of any of the monitoring sensors(antecedents) change, we refer back to the rule, deactivate it and return the activated sensor(s)(consequents) to regular operation.

\section{IMPLEMENTATION AND RESULTS}

To validate our methodology, we perform a simulation run for the lifetime of a hypothetical query using a $\mathrm{C}$ implementation of the algorithm we proposed, with synthetic sensor data transactions piped to the program. The simulation is done on a $\mathrm{PC}$, assuming that we have a node $\mathrm{M}$ that collects light, temperature and microphone readings from three other sensor streams coming from sensors $\mathrm{S} 0$, $\mathrm{S} 1$ and S2. Two generic types of queries are examined: (i) the worst case scenario when a query requests for all sensory values from $\mathrm{M}$, and (ii) the best case scenario when the query only requests for a select number of values from $\mathrm{M}$. The measure for data transmitted shows the amount of radio communication we can reduce; radio communication being the highest resource drain factor for sensors. As an overview, in the following experiments, the first 8 minutes run is used to obtain the rules from the correlated data using our algorithm and when the query is posted, these gathered rules are used for the query at subsequent runtimes. But first, we estimate the amount of data we can reduce by using this algorithm when the query:

\section{SELECT * FROM sensors, SAMPLE PERIOD 1s FOR 7 minutes}

is posted to node $M$. In this scenario, consider that each sensor has three attributes (light $\mathrm{l}$, temperature $\mathrm{t}$, microphone $\mathrm{m}$ ) - so that we have $\left(l_{1}, t_{1}, z_{1}\right)$ for the first sensor and so on. The synthetic data that we have generated has the attribute that: (1) So light readings and S1 light readings have a positive correlation of $0.8+-0.04$ (2) S1 light readings and $\mathrm{S} 1$ temperature readings have a positive correlation of $0.8+/-0.04$ (3) S2 light readings and S2 temperature readings have a negative correlation of $-0.8+-0.04$.

\subsection{Evaluation of Data Throughput for Query with Rule Adaptation}

Table 1 shows the rules obtained when the algorithm runs on our synthetic dataset for 8 minutes. The column on success rate shows the percentage of packets that have been correctly predicted out of all the data transactions that holds the same antecedent values from the applied rules. From the table, we observe that the rules that we get capture the correlation patterns generated in the data, for example $S 1$ Light $\rightarrow$ S0 Light, which are also high confidence rules. After a runtime of 8 minutes, the algorithm stores the rules collected in a rule queue. When node $M$ is required to obtain sensor readings from sensors S0, S1 and S2, it will first look at all the
Table 1: Table of Rules Discovered in Simulation Run

\begin{tabular}{|c|c|c|c|c|}
\hline \multicolumn{3}{|c|}{ Discovered Rules } & \multicolumn{2}{|c|}{ 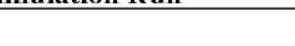 } \\
\hline Runtime & RuleID & Rules & Conf. & SuccessRate $\%$ \\
\hline 1 & R1 & $S 1 L[H] \rightarrow S 0 L[H]$ & 0.83 & $82.9 \%(34 / 41)$ \\
\hline 1 & R2 & $S 0 L[H] \rightarrow S 1 L[H]$ & 1.0 & $100 \%(34 / 34)$ \\
\hline 3 & R3 & $S 1 L[H] \rightarrow S 0 L[H]$ & 0.83 & $82.9 \%$ \\
\hline 3 & R4 & $S 0 L[H] \rightarrow S 1 L[H]$ & 1.0 & $100 \%$ \\
\hline 3 & R5 & $S 0 M[Y] \rightarrow S 0 L[L]$ & 0.5 & $45.3 \%(24 / 53)$ \\
\hline 3 & R6 & $S 0 L[L] \rightarrow S O M[Y]$ & 1.0 & $85.7 \%(24 / 28)$ \\
\hline 5 & R7 & $S 1 T[L] \rightarrow S 0 L[H]$ & 1.0 & $100 \%(20 / 20)$ \\
\hline 5 & R8 & $S 0 L[H] \rightarrow S 1 T[L]$ & 0.73 & $58.9 \%(20 / 34)$ \\
\hline 7 & R9 & $S 1 T[L] \rightarrow S 0 L[H]$ & 1.0 & $100 \%$ \\
\hline 7 & R10 & $S 0 L[H] \rightarrow S 1 T[L]$ & 0.64 & $58.9 \%$ \\
\hline
\end{tabular}

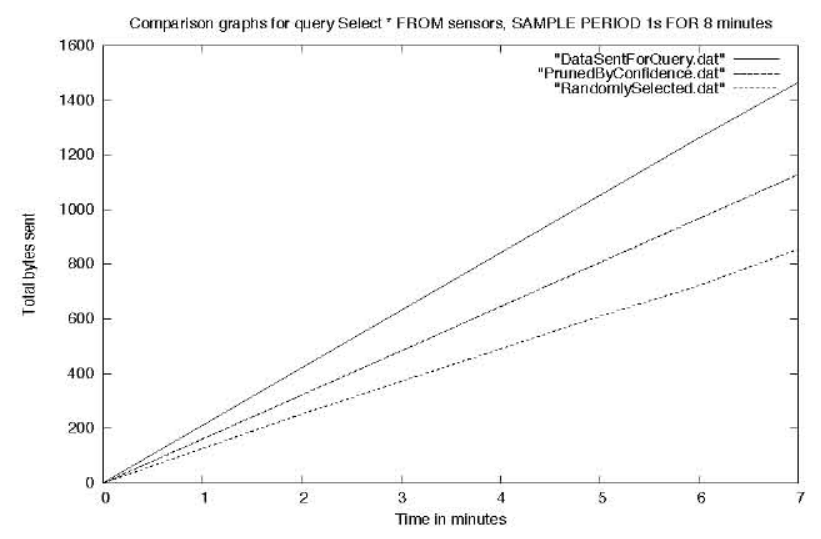

Figure 1: Comparison graphs for query Select* FROM sensors, SAMPLE PERIOD 1s FOR 7 minutes

rules that have been stored in the rule queue. If a rule can be applied, then $M$ will just infer based on the rule, the reading of the implied sensor while the antecedent sensor of the rule is being monitored. Currently, the basic pruning strategy that we use on the rules is such that they must have a minimum confidence of 0.8 to be considered useful(in algorithm, only threshold confidence of 0.3 set). The threshold is also used to imply that a rule no longer becomes active if the antecedent of the rule shows a differing discrete state than anticipated more than $20 \%(1-$ minimumConfidence $* 100)$ of the time or in 2 transactions out of 10 . For this query, we select R6 and R7 because they are high confidence unique rules in the rule queue that can be used by a query processor to conserve energy while answering this query. In Table 1, note that some rules which conflict with earlier rules are not used. An example of conflicting rules are R1 and R2, where if you use R1(S1's high light reading to predict SO's high light reading), you can't use R2 because you will need S1 to be active in order to monitor S0. Rules which share this type of relationship are, therefore, in conflict with

Table 2: Rule Pruned for Specific query

\begin{tabular}{r|rrrr}
\hline \multicolumn{5}{c|}{ Recorded Data Transmitted and Errors (in Bytes) } \\
\hline \hline RuleUsed & Conf. & PacketsSent & TotalErrors & ActualSaved \\
Selected R4 & $1.0 \%$ & 0 & 0 & 238 \\
Random R8 & 0.73 & 238 & - & 0 \\
No Rule & - & & 0 & 0 \\
\hline
\end{tabular}


one another. One way we can resolve this conflict is that, for a new rule, if the antecedent of the new rule is the consequent of earlier active rules before it, it won't be made active.

Figure 1 shows the amount of bytes you can reduce when you can query less sensors by applying our associations rules for this particular query. In the plot corresponding to "PrunedByConfidence.dat", rules R6 and R7 have been used to filter the data, and this gives a success rate of $91.7 \%$ ( 4 errors out of 48 predicted). In comparison, while the third plot using randomly selected rules R8 and R5 saves more transmission costs, the success rate of the prediction is only $50.6 \%$ (43 errors out of 87 predicted). We do, however, believe that the pruning strategy can be further improved as some useful rules have been neglected due to conflicts and we notice that, if we put a heavy emphasis on sensors in the antecedent of the rules, a failure in those sensor can mean that some rules can be turned on indefinitely, giving us false readings. We are currently still working on developing a better pruning strategy for our algorithm and porting the algorithm to berkeley motes. One other aspect to improve the algorithm could be to select only antecedents of the rule with a high support so that a rule can be valid for more transactions.

Table 2 shows the effect of using non-conflicting rules based on the current query. In this instance, the query

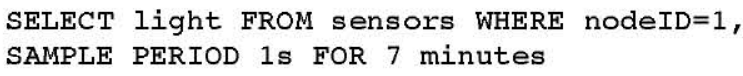

allows us to choose only the rule that has consequent sensor with nodeid 1. Here, only R4 is selected because from the set of rules available, only R4 is able to predict the value of S1 with a high confidence. Any other random rule that would be used then has no effect on energy saved.

\section{CONCLUSION AND FUTURE WORK}

To summarise, we have presented in this paper, a light-weight rule learning algorithm to mine for highly correlated rules in resourceconstrained sensor networks, which we believe would enable us to conserve energy with rule triggers efficiently. For sensor groupings, the two main parts of any rule allows us to control some sensors(consequent of rule) while the antecedent of rule is used to monitor that the rule has changed. On the other hand, rules can also be used to infer readings of sensors and here, we have shown that we can reduce the number of packets that needs to be transmitted with a query with rule adaptation. As shown in $\S 2$, although several authors have looked at performing data processing on sensor nodes, none have looked at discovering rules on sensors, whereby running a centralised rule mining algorithm such as APRIORI would be infeasible. We believe that the application of rule learning on sensors can bring significant energy savings as it would enable triggers to conserve energy on sensors when sensors are not required to actively sample data or perform sensing at all. In the future, we wish to further examine the algorithm in light of how it would perform in a group setting with real motes and also, manipulate the other rule triggers. Another interesting issue to explore is rule prioritisation and determining a formal method to score each rule based on how much energy we can save or how critical the sensing is required.

\section{REFERENCES}

[1] MICA2 Mote Datasheet, http : //www.xbow.com/Products/Product_pdf -files/Wireless_pdf/6020 - 0042-05 A_MICA2.pdf (Accessed 08/06/2006)
[2] Rakesh Agrawal, and Ramakrishnan Srikant, Fast Algorithms for Mining Association Rules, Proceedings of the 20th VLDB, pp. 487-499, 1994.

[3] I. Akyildiz, W. Su, Y. Sankarasubramaniam, and E. Cayirci, Wireless Sensor Networks: A Survey, Computer Networks, Vol. 38 , pp. 393-422, 2002.

[4] G. Asada, T. Dong, F. Lin, G. Pottie, W. Kaiser, and H. Marcy, Wireless Integrated Network Sensors: Low Power Systems on a Chip, European Solid State Circuits Conference, The Hague, Netherland, 1998.

[5] A.P. Chandrakasan, S. Sheng and R.W. Brodersen, Low Power CMOS Digital Design, IEEE Journal of Solid-State Circuits, Vol. 27, pp. 473-484, 1992.

[6] S.K. Chong and S. Krishnaswamy and S.W. Loke, A Context-Aware Approach to Conserving Energy in Wireless Sensor Networks, Proceedings of the First International Workshop on Sensor Networks and Systems for Pervasive Computing, Hawaii, 2005.

[7] A. Demers, J. Gehrke, R. Rajaraman, N. Trigoni, and Y. Yao, Energy-Efficient Data Management for Sensor Networks: A Work-In-Progress Report, 2004.

[8] E. Elnahrawy, and B. Nath, Context-Aware Sensors, Proceeedings of the 1st European Workshop on Wireless Sensor Networks, pp.77-93, Springer-Verlag, Berlin, Heidelberg, 2004.

[9] M. Galan, H. Liu, and K. Torkkola, Intelligent Instance Selection of Data Streams for Smart Sensor Applications, AGLT05, 2005.

[10] C. Guo, L.C. Zhong and J.M. Rabaey, Low Power Distributed MAC for Ad Hoc Sensor Radio Networks, Global Telecommunications Conference, Vol. 5, pp. 25-29, 2001.

[11] K.K. Loo, I. Tong, B. Kao, and D. Cheung, Online Algorithms for Mining Inter-Stream Associations From Large Sensor Networks, PAKDD, pp. 143-149, 2005.

[12] M. XiuLi, Y. Dongqing, T. Shiwei, L. Qiong, D. Zhang, L. Shuangfeng, Online Mining in Sensor Neworks, LNCS 3222, pp. 544-550, 2004.

[13] S. Madden, and M.J. Franklin, Fjording the Stream: An Architecture for Queries over Streaming Sensor Data, Proceedings of the 18th International Conference on Data Engineering, pp. 55, California, 2002.

[14] R. Marin-Perianu, M. Marin-Perianu, and P. Havinga, Movement-based Group Awareness with Wireless Sensor Networks, Proceedings of Pervasive 2007, 2007.

[15] P. Radivojac, U. Korad, K.M. Sivalingam, and Z. Obradovic, Learning from Class-Imbalanced data in Wireless Sensor Networks, RKS05, 2005.

[16] V. Raghunathan, C. Schurgers, S. Park, and M.B. Srivastava, Energy Aware Wireless Microsensor Networks, IEEE Signal Processing Magazine, Vol. 19, pp. 40-50, 2002.

[17] M. Younis and P. Munshi and E. Al-Shaer, Architecture for Efficient Monitoring and Management of Sensor Networks, Management of Multimedia Networks and Services, $6 \mathrm{th}$ IFIP/IEEE International Conference MMNS 2003, pp. 488-502, Springer, Belfast, UK, 2003.

[18] L.Yuan and G. Qu, Design Space Exploration for Energy-Efficient Secure Sensor Network, Proceedings of the IEEE International Conference on Application-Specific Systems, Architectures, and Processors, pp. 1-10, San-Jose, California, 2002. 